\section{A mouse GM-CSF receptor antibody attenuates neutrophilia in mice exposed} to cigarette smoke

\author{
F.M. Botelho*, J.K. Nikota*, C. Bauer", N.H.E. Davis", E.S. Cohen", I.K. Anderson", \\ A.J. Coyle ${ }^{+}$, R. Kolbeck ${ }^{+}$, A.A. Humbles ${ }^{+}$, M.R. Stämpfli ${ }^{*},{ }^{\xi}, f$ and M.A. Sleeman ${ }^{\uparrow, f}$
}

ABSTRACT: We investigated the role of granulocyte-macrophage colony-stimulating factor (GMCSF) in a subchronic exposure model of cigarette smoke (CS)-induced inflammation using antibodies directed against GM-CSF or the GM-CSF receptor (GM-CSFR) $\alpha$-chain.

CS-induced mononuclear and neutrophilic inflammation following 4 days of CS exposure in BALB/c mice was assessed in bronchoalveolar lavage (BAL) fluid. An increase in mature dendritic cells (DCs) (CD11c+ and major histocompatibility complex II+) and Gr-1-high neutrophils was also observed by flow cytometric analysis of whole-lung tissue.

Daily i.p. injection of $400 \mu \mathrm{g}$ GM-CSF or GM-CSFR antibody prior to daily smoke exposure attenuated the accumulation of neutrophils within the BAL by $60 \%$. A reduction in mature DCs was also observed. Anti-GM-CSFR antibody administration did not have an effect on the percentage of lung T-cells; however, a significant decrease in activated CD69+ CD8+ T-cells was observed. AntiGM-CSFR antibody administration decreased the mRNA and protein expression of interleukin-12 p40 and matrix metalloproteinase 12.

Taken together, intervention with this receptor antibody implicates the GM-CSF pathway as an important mediator of smoke-induced inflammation.

KEYWORDS: Antibody neutralisation, cigarette smoke, granulocyte-macrophage colony-stimulating factor, inflammation, neutrophils

W hilst lung exposure to cigarette smoke (CS) has been identified as the leading cause of chronic obstructive pulmonary disease (COPD), the exact pathogenic mechanisms of the disease are not understood [1]. COPD is characterised by irreversible airflow obstruction and progressive lung inflammation that correlates with disease severity [2]. Whilst asthma is more associated with eosinophilic inflammation, in COPD, neutrophils and macrophages predominate [3].

As a key regulator of myeloid cell survival and activation [4], the cytokine granulocyte-macrophage colony-stimulating factor (GM-CSF) has been shown to have a central role in maintaining the innate immune response in the healthy lung [ 5 , 6]. It is released by a range of structural and inflammatory cells, such as the airway epithelium, smooth muscle cells, T-cells and macrophages. It has also been shown that GM-CSF is elevated in the lungs of patients with COPD [7-9], suggesting that this cytokine may also play a role in the exaggerated inflammatory response in the disease. The contribution of GM-CSF is unclear; however, it has been shown that GM-CSF not only activates macrophages and neutrophils in concert with stimuli, such as lipopolysaccharide, that may be present in the COPD lung, but also promotes cytokine release and cell survival [2]. Recently, it has also been demonstrated that intranasal delivery of an anti-GM-CSF antibody in a mouse model of CS-induced inflammation attenuates lung neutrophilia [10].

GM-CSF mediates its effects via a GM-CSF receptor (GM-CSFR) that consists of two subunits, an $\alpha$-subunit, which binds the cytokine with low affinity, and a larger $\beta$-subunit (beta common; $\beta c$ ), responsible for signalling, forming a ternary receptor complex [11]. Signal transduction in response to the cytokines interleukin (IL)- 3 and IL-5 is also mediated by $\beta c$; therefore, receptor specificity is due to GM-CSFR $\alpha$ [12].

This article has supplementary material available from www.erj.ersjournals.com

AFFILIATIONS

*Dept of Pathology and Molecular Medicine, Centre for Gene Therapeutics,

${ }^{*}$ Medical Sciences Graduate Program and

${ }^{\S}$ Dept of Medicine, McMaster University, Hamilton, ON, Canada. "Dept of Respiratory, Autoimmunity and Inflammation, Medlmmune LLC, Cambridge, UK.

${ }^{+}$Dept of Respiratory, Autoimmunity and Inflammation, MedImmune LLC, Gaithersburg, MD, USA.

${ }^{\text {f}}$ Both authors contributed equally to the design of these studies.

CORRESPONDENCE

M.A. Sleeman

Medlmmune

Milstein Building

Granta Park

Cambridge

CB21 6GH

UK

E-mail: sleemanm@

medimmune.com

Received:

May 142010

Accepted after revision:

Feb 022011

First published online:

March 242011 

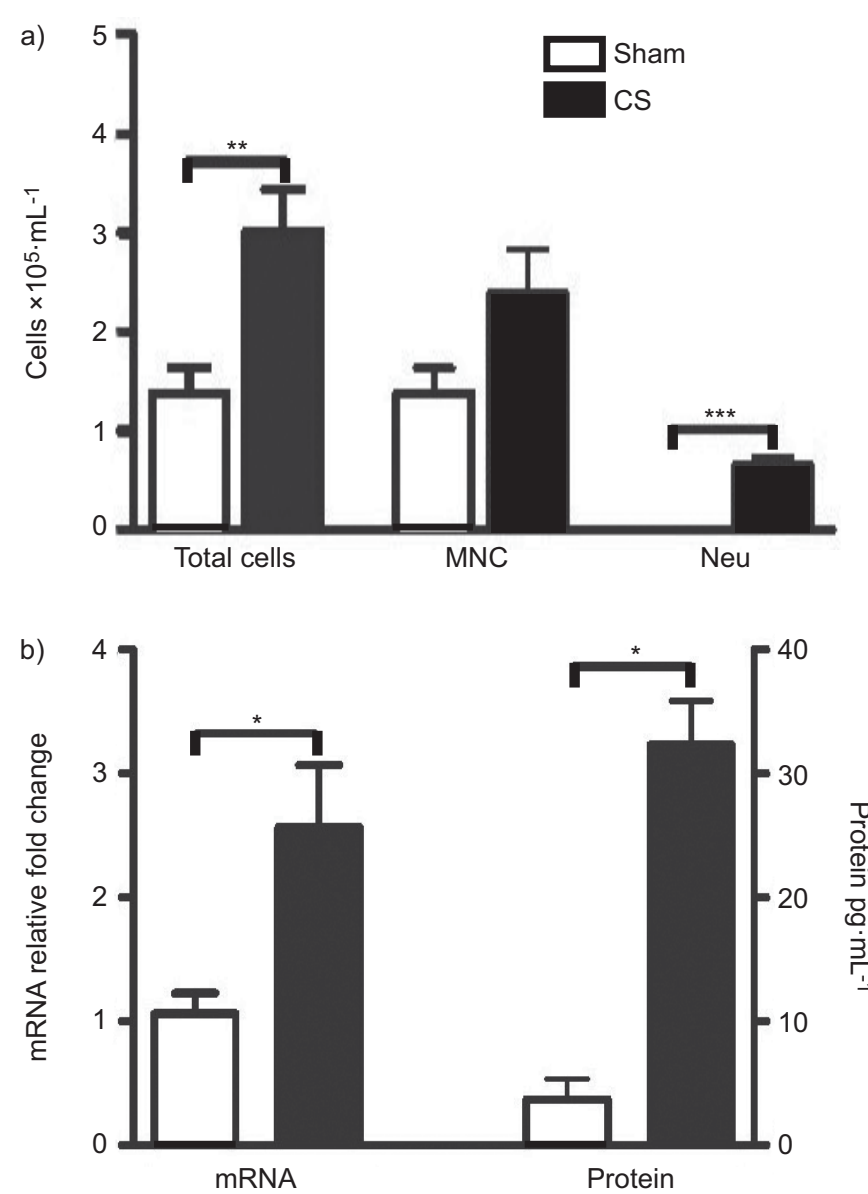

FIGURE 1. Bronchoalveolar lavage (BAL) cellular profile and granulocytemacrophage colony-stimulating factor (GM-CSF) expression in cigarette smoke (CS)-exposed mice. BALB/c mice were sham- or CS-exposed for 4 days. a) BAL total cell number, mononuclear cells (MNC) and neutrophils (Neu). $n=5$ per group. b) GM-CSF mRNA (sham: $n=3 ; C S: n=8$ ) and protein expression ( $n=5$ per group) in the lungs of sham- and CS-exposed mice. Statistical analysis was performed using the nonparametric Kruskall-Wallis test with a Mann-Whitney post-test. Bars represent means and whiskers represent standard errors of the mean. ${ }^{*}: p<0.05$; $* *: p<0.01 ; * * *: p<0.001$.

Because CS is one of the main causative agents of COPD and a number of CS-exposure models have been developed that present with phenotypes analogous to COPD, such as emphysema and lung inflammation [13-15], these models provide platforms to evaluate the efficacy and mechanisms of new therapies. Biologics are increasingly being developed for chronic diseases, such as tumour necrosis factor (TNF)- $\alpha$ antibodies and receptor antagonists in arthritis [16-18], and anti-immunoglobulin (Ig)E antibodies in asthma [19]. Moreover, antibody trials on COPD are also underway that target IL-1 $\beta$ (canakinumab) [20]. Currently a number of anti-GM-CSF and anti-GM-CSFR $\alpha$ [4] approaches are in early clinical development for rheumatoid arthritis. To date, only a single study has been conducted investigating the role of GM-CSF in a CS-exposure model via intranasal delivery [10]. In the present study, we continued this work by evaluating a neutralising antibody to GM-CSF (22E9) and a GM-CSFR $\alpha$ (CAM-3003) antibody in the subchronic CS model dosed via the systemic
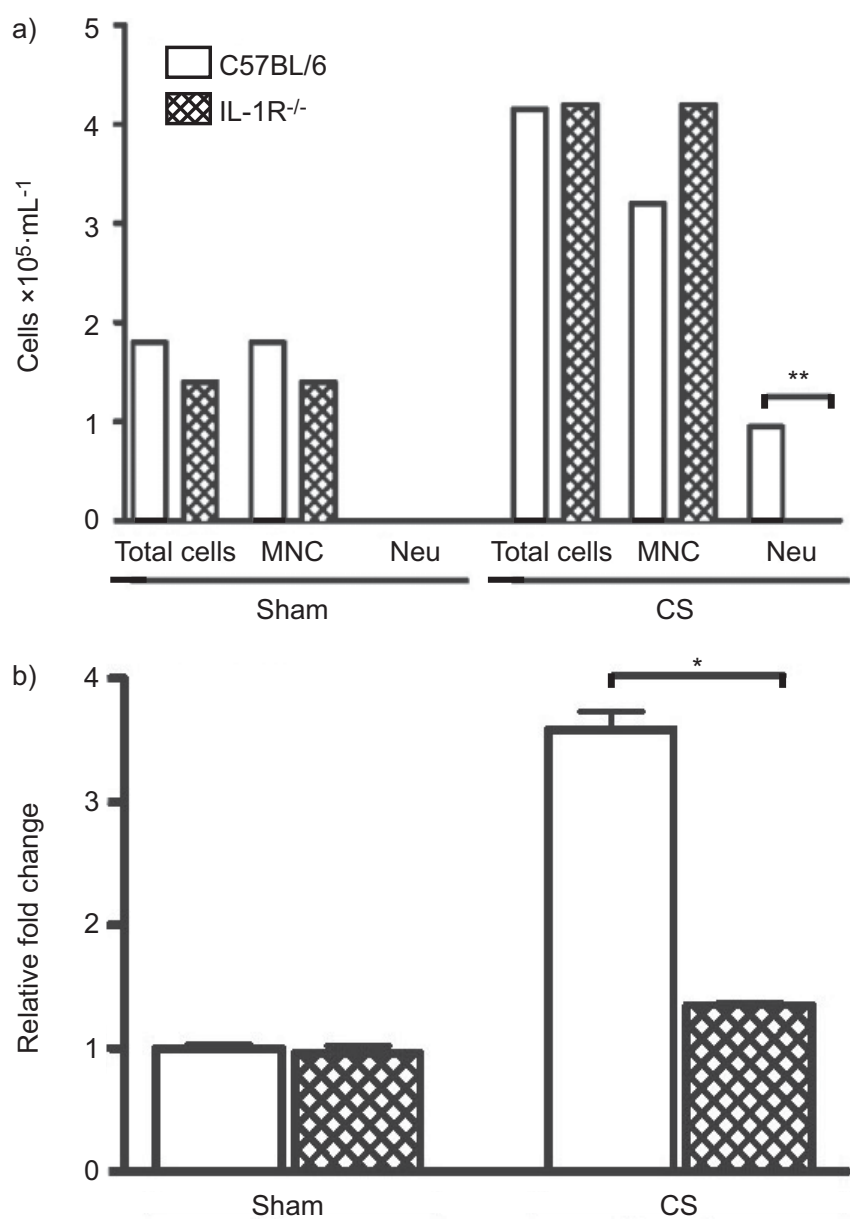

FIGURE 2. Bronchoalveolar lavage (BAL) cellular profile and granulocytemacrophage colony-stimulating factor (GM-CSF) expression in cigarette smoke (CS)-exposed interleukin-1 receptor (IL-1R) knockout mice. a) Wild-type and IL-1 $\mathrm{R}^{-1-}$ mice were exposed to room air (sham) or CS for 4 days. BAL total cell number, mononuclear cells (MNC) and neutrophils (Neu) are shown. b) GM-CSF mRNA

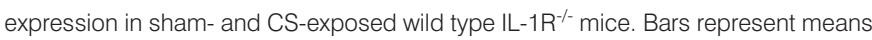
and whiskers represent standard errors of the mean. $n=5$ per group. Statistical analysis was performed using two-way ANOVA with a Bonferroni post-test for wildtype versus knockout. *: $p<0.05 ;{ }^{*}: p<0.01$.

compartment to determine the effects of inhibiting this pathway, via a more clinically relevant route of antibody administration. Moreover, we also explored the role of GM-CSF pathway blockade on downstream cytokines, and its effects on dendritic cell (DC) subsets in the lung and on CD8+ T-cell activation.

\section{MATERIALS AND METHODS Animals}

Female BALB/c mice (6-8 weeks of age) were purchased from Charles River Laboratories (Montreal, QC, Canada). IL-1 receptor knockout and wild-type (WT) control C57BL/6 mice were obtained from Jackson Laboratory (Bar Harbor, ME, USA). Mice were maintained under specific pathogen-free conditions, on a 12-h light-dark cycle, with food and water provided ad libitum. The Animal Research Ethics Board of McMaster University (Hamilton, ON, Canada) approved all experiments. 

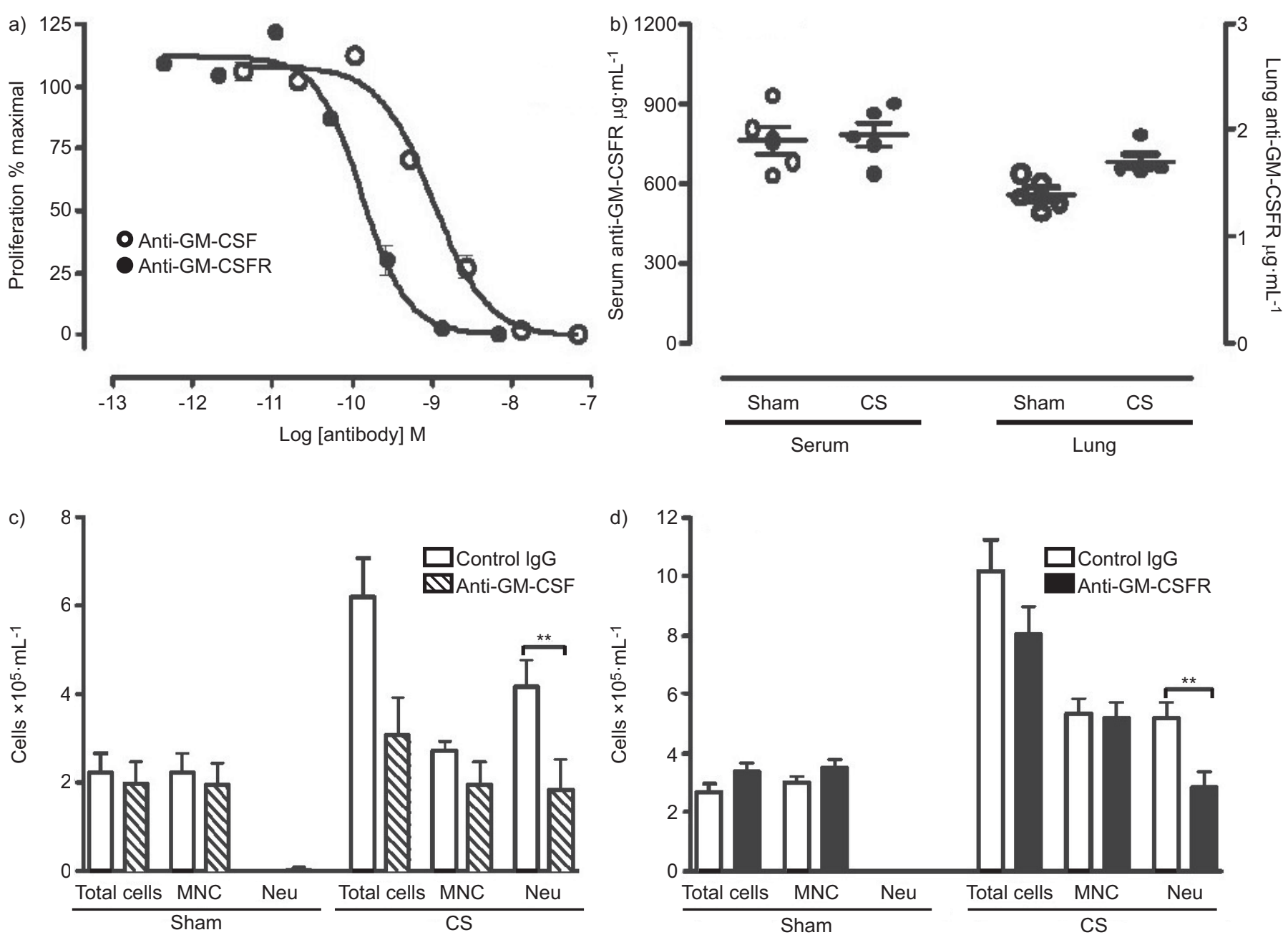

FIGURE 3. Granulocyte-macrophage colony-stimulating factor (GM-CSF) neutralising activity and the bronchoalveolar lavage cellular profile in cigarette smoke (CS)exposed mice injected with anti-GM-CSF ligand or anti-GM-CSF receptor (anti-GM-CSFR) antibodies. a) Potency comparison of anti-GM-CSF and anti-GM-CSFR in a mouse GM-CSF FDCP-1 proliferation assay. Cells were incubated with mouse GM-CSF and a dilution series of antibody for $16 \mathrm{~h}$. Cell proliferation was quantified following a further $4 \mathrm{~h}$ incubation with tritiated thymidine. b) Terminal exposure of anti-GM-CSFR levels in serum and lung homogenates from sham- and CS-exposed mice. BALB/c mice were exposed to CS for 4 days. Mice were injected i.p. with either c) anti-GM-CSF ligand or d) anti-GM-CSFR, or isotype control antibodies $18 \mathrm{~h}$ prior to daily CS exposures. Data show total cell numbers, mononuclear cells (MNC) and neutrophils (Neu). Bars represent means and whiskers represent standard errors of the mean. $\mathrm{n}=5$ per group. Statistical analysis was performed using a two-way ANOVA with a Bonferroni post-test for sham versus CS groups. **: $p<0.01$.

\section{FDCP-1 cell proliferation assay}

FDCP-1 cells were maintained in Dulbecco's modified Eagle's medium (Invitrogen, Burlington, ON, Canada) containing 10\% fetal bovine serum (FBS; JRH Biosciences, Lenexa, KS, USA) and $5 \mathrm{ng} \cdot \mathrm{mL}^{-1}$ murine GM-CSF (R\&D Systems, Minneapolis, MN, USA). Prior to the assay, cells were cultured in the absence of GM-CSF for $30 \mathrm{~h}$ at $37^{\circ} \mathrm{C}$ in $5 \% \mathrm{CO} 2$. Cells were resuspended at $1.5 \times 10^{5}$ cells $\cdot \mathrm{mL}^{-1}$ in medium and $100 \mu \mathrm{L}$ of this suspension was added to each well of a flat-bottomed, 96-well plate. Cells were incubated at $37^{\circ} \mathrm{C}$ in $5 \% \mathrm{CO} 2$ with antibody in the presence of 1-2 pM murine GM-CSF for $16 \mathrm{~h}$. $20 \mu \mathrm{L}$ tritiated thymidine $\left(5.0 \mathrm{mCi} \cdot \mathrm{mL}^{-1}\right)$ was added to each well and incubated for $4 \mathrm{~h}$ prior to harvesting on GF/C Unifilter ${ }^{\mathrm{TM}}$ plates (Perkin Elmer, Woodbridge, ON, Canada). Thymidine incorporation was determined using a scintillation counter (Packard Topcount; Packard Instrument Company, Meriden, CT, USA). Data were analysed using Prism software (GraphPad, San Diego, CA, USA).

\section{CS-exposure protocol}

Mice were exposed to CS from 12 2R4F reference cigarettes with filters removed (Tobacco and Health Research Institute, Lexington, KY, USA) for $\sim 50 \mathrm{~min}$, twice daily, for 4 days using a whole body smoke exposure system (SIU-48; Promech Lab AB, Vintrie, Sweden) as described previously [21]. CS exposure followed an initial acclimatisation period whereby on day 1 , mice were placed into the exposure box for $20 \mathrm{~min}$, on day 2, for $30 \mathrm{~min}$ and on day 3, for $50 \mathrm{~min}$. Control animals (sham), were exposed to room air only.

\section{i.p. administration of antibodies}

BALB/c mice were injected i.p. with $400 \mu \mathrm{g}$ anti-GM-CSFR (CAM-3003; mouse IgG1) or anti-GM-CSF ligand (MP122E9 clone; rat IgG2a; R\&D Systems) antibody $12 \mathrm{~h}$ prior to each of the first daily CS exposures. Mice were sacrificed 12-18 h after the last CS exposure. 


\section{Bronchoalveolar lavage and differential cell counting}

Bronchoalveolar lavage (BAL) fluid was collected as follows. Briefly, lungs were dissected, cannulated and instilled with $0.25 \mathrm{~mL}$ ice-cold $1 \times$ PBS, followed by $0.2 \mathrm{~mL} 1 \times$ PBS. Total cell numbers were counted using a haemocytometer. Cytospins were prepared for differential cell counts and stained with Hema 3 (Biochemical Sciences Inc., Swedesboro, NJ, USA). 500 cells were counted per cytospin to identify mononuclear cells, neutrophils and eosinophils.

\section{ELISA and meso-scale discovery analysis}

ELISA kits for GM-CSF were purchased from R\&D Systems and used according to manufacturer's protocol. Cytokine detection of interferon (IFN)- $\gamma$, IL-1 $\beta$, IL-4, keratinocytederived cytokine $(\mathrm{KC})$ and IL-12 was performed using the multi-array murine pro-inflammatory and T-helper cell (Th) type 1 /Th2 cytokine panel detection system (MesoScaleDevices, Gaithersburg, MD, USA).

\section{mRNA expression Fluidigm analysis}

RNA was extracted from a single lobe using the Qiagen RNeasy Fibrous Tissue kit according to manufacturer's
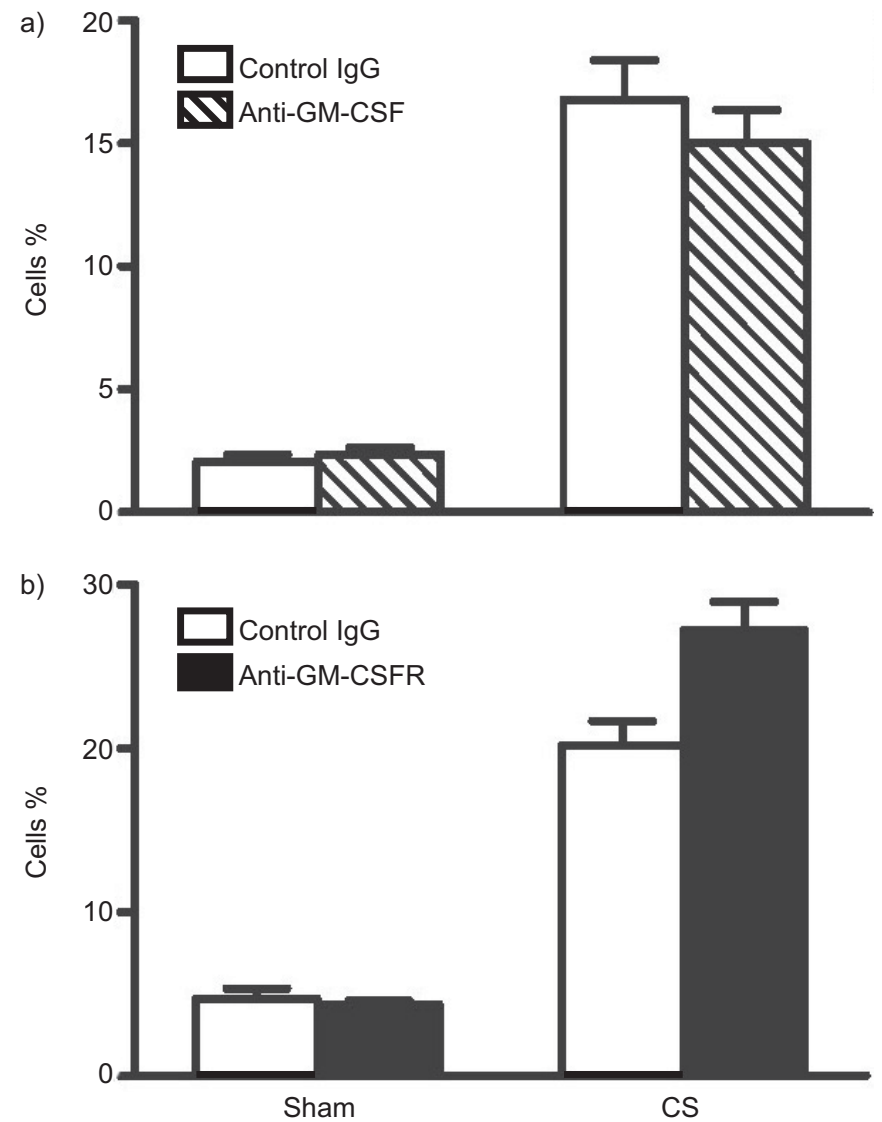

FIGURE 4. Flow cytometric analysis of neutrophils (Gr-1-high) in sham and cigarette smoke (CS)-exposed mice injected with anti-granulocyte-macrophage colony-stimulating factor (GM-CSF) ligand or anti-GM-CSF receptor (GM-CSFR) antibodies. BALB/c mice were exposed to CS for 4 days. Mice were injected i.p. with either a) anti-GM-CSF or b) anti-GM-CSFR antibodies, or isotype control antibodies. Neutrophils (Gr-1-high, major histocompatibility complex class II-high) were examined in whole-lung, single-cell suspensions by flow cytometry. Statistical analysis was performed using two-way ANOVA with a Bonferroni post-test for sham versus smoke. protocol (Qiagen, Hilden, Germany). RNA was quantified and normalised, and integrity assessed using the Agilent RNA 6000 Nano Kit (Agilent, Santa Clara, CA, USA). cDNA generation was carried out with the Super Script III kit (Life Technologies, Carlsbard, CA, USA). Relative transcript expression was assessed using the Fluidigm Biomark Dynamic Array (Fluidigm, Amsterdam, the Netherlands) loaded with probes for transcripts of interest.

\section{Isolation of lung mononuclear cells}

Lung mononuclear cells were isolated as described previously [21]. Briefly, lungs were perfused with $1 \times$ PBS, and cell suspensions were generated by disaggregation and incubation for $1 \mathrm{~h}$ at $37^{\circ} \mathrm{C}$ in $150 \mathrm{U} \cdot \mathrm{mL}^{-1}$ collagenase III in hydroxyethyl piperazine ethane sulfonic acid-buffered saline solution (Invitrogen). Debris was removed by passing through a nylon mesh. Cells were resuspended in $1 \times$ PBS containing $0.3 \%$ bovine serum albumin (BSA) (Invitrogen) or in RPMI supplemented with 10\% FBS (Sigma-Aldrich, Oakville, ON, Canada), $1 \%$ L-glutamine and 1\% penicillin/streptomycin (Invitrogen).

\section{Flow cytometric analysis}

$1 \times 10^{6}$ lung mononuclear cells were washed once with $1 \times$ PBS/ $0.3 \%$ BSA and stained with directly conjugated primary antibodies for $30 \mathrm{~min}$ at $4^{\circ} \mathrm{C} .10^{5}$ live events were acquired using an LSR II (BD Biosciences, Mississauga, ON, Canada) flow cytometer and analysed with FlowJo software (TreeStar Inc., Ashland, OR, USA). The following antibodies were used: fluorescein isothiocyanate-conjugated anti-CD11c, phycoerythrin (PE)-conjugated anti-CD11b, PE-Alexa Fluor 610-conjugated CD8, PE-cy5.5-conjugated anti-CD19, PE-cy7-conjugated anti-CD69, allophycocyanin (APC)-conjugated anti-major histocompatibility complex (MHC) class II, Alexa Fluor 700conjugated anti-CD86, APC-cy7-conjugated anti-CD45, Pacific Blue-conjugated anti-CD3 and Pacific Orange-conjugated antiGr-1. These antibodies were purchased from BD Biosciences (San Jose, CA, USA) or eBioscience (San Diego, CA, USA) except for CD86 (BioLegend, San Diego, CA, USA). Quantum dot (Qdot)605-conjugated anti-CD4 and Qdot655-conjugated antiB220 were purchased from Invitrogen (Carlsbad, CA, USA).

\section{Statistical analysis}

Data were analysed using Prism Software version 5 (Graphpad) and expressed as mean \pm SEM. Statistical analysis was performed by testing for a normal distribution using the Kolmogorov-Smirnov test followed by an unpaired t-test, oneway ANOVA or a two-way ANOVA with a Bonferroni posttest, as stated in figures. Nonparametric tests were undertaken using the Kruskall-Wallis test with a Mann-Whitney post-test. Differences were considered statistically significant when $\mathrm{p}$ values were $<0.05$.

\section{RESULTS}

\section{CS-induced inflammation is associated with increased GM- CSF $m R N A$ and protein expression}

In order to investigate the impact of CS exposure on GM-CSF expression in the lungs, we exposed BALB/c mice to CS for 4 days. Control animals were exposed to room air only (sham). CS exposure led to a significant increase in total cell numbers in BAL, which predominantly reflected neutrophils (fig. 1a). CS significantly increased the levels of GM-CSF in the lung (fig. 1b). 

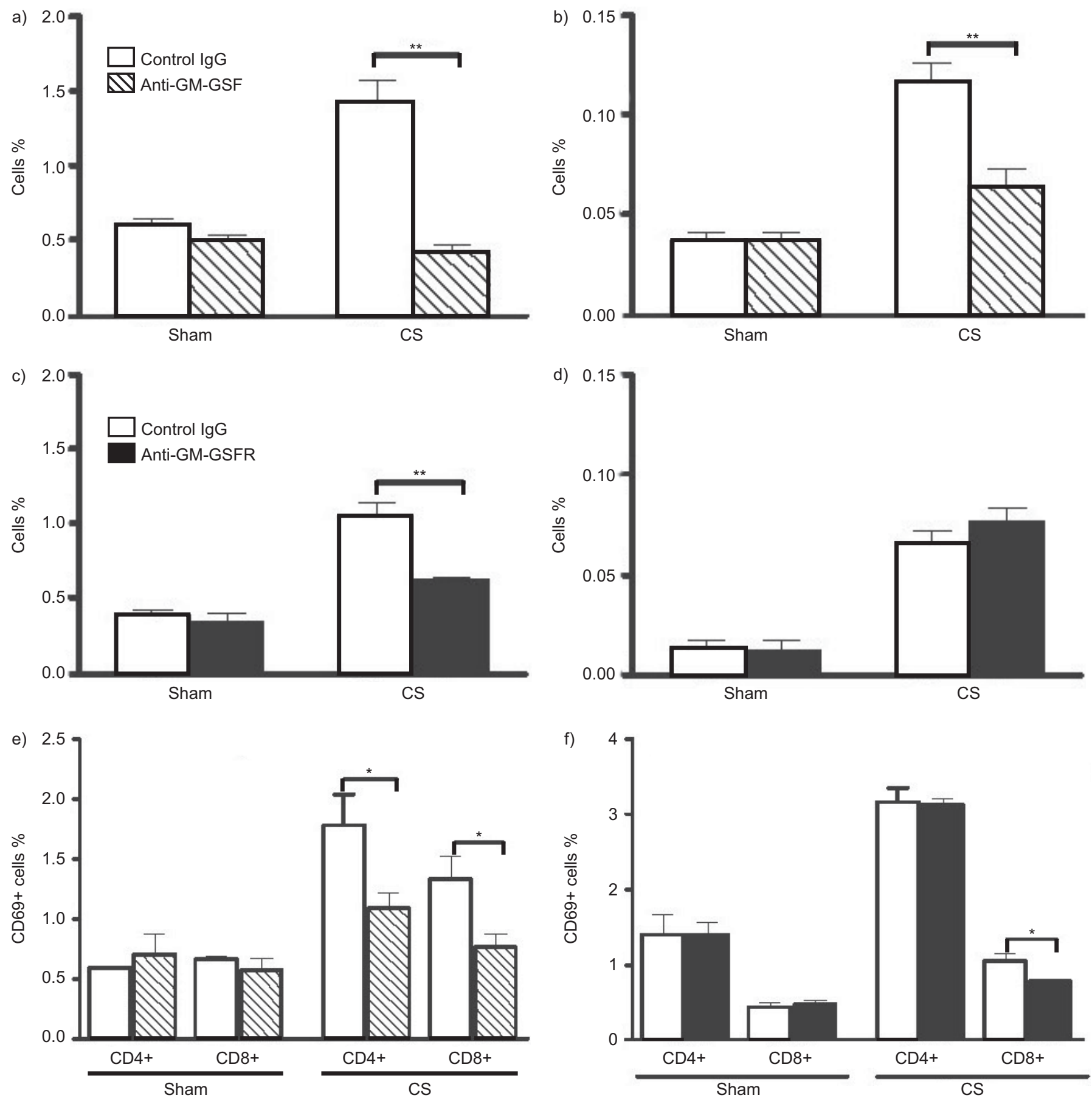

FIGURE 5. Flow cytometric analysis of dendritic cell (DC) subsets and activated T-cells in cigarette smoke (CS)-exposed mice injected with anti-granulocytemacrophage colony-stimulating factor (GM-CSF) ligand or anti-GM-CSF receptor (GM-CSFR) antibodies. BALB/c mice were exposed to CS for 4 days. Mice were injected i.p. with either a, b, e) anti-GM-CSF ligand antibodies or c, d, f) anti-GM-CSFR antibodies, or isotype control antibodies. a, c) Myeloid DCs (CD11c-high, major histocompatibility complex (MHC) class II-high B220-) and b, d) plasmacytoid DCs (CD11c-high, MHC II-high B220+), or e, f) activated CD69+ CD4+ and CD8+ T-cell subsets were examined in whole-lung single-cell suspensions by flow cytometry. Statistical analysis was performed using a two-way ANOVA with a Bonferroni post-test for sham versus CS. Ig: immunoglobulin. *: $p<0.05 ; * *: p<0.01$.

\section{Mechanisms of GM-CSF expression}

Although multiple pathways probably contribute to CSinduced inflammation, we next sought to investigate whether expression of GM-CSF following smoke exposure was IL-1 receptor (IL-1R)1-dependent. This experiment was based on a recent report by Doz et al. [22] demonstrating an important role of IL-1R1 in CS-induced inflammation. Consistent with the findings of Doz et al. [22], CS-induced neutrophilia was markedly attenuated in IL-1R-deficient mice (fig. 2a). Loss of IL-1R expression did not significantly affect the increase in mononuclear cells following CS exposure. We further examined GM-CSF mRNA expression in C57BL/6 (WT) and 
IL-1R-deficient mice that were either sham or CS exposed. GMCSF mRNA expression was abrogated in the IL-1R-deficient mice (fig. 2b).

\section{Anti-GM-CSF ligand and receptor antibodies attenuate CS- induced lung inflammation}

In order to test the importance of GM-CSF to CS-induced airway inflammation, we administered either anti-ligand (22E9) or receptor-blocking antibodies (CAM-3003) to shamor CS-exposed mice. The activity of each antibody was confirmed in the FDCP-1 proliferation assay (fig. 3a). The median inhibitory concentration of 22E9 and CAM-3003 was $919 \mathrm{pM}$ (95\% CI 694-1,218 pM) and $130 \mathrm{pM}$ (95\% CI 99$171 \mathrm{pM})$, respectively. Due to the different potencies and isotypes of these antibodies, fully saturating doses of each antibody were used in these studies. Pharmacokinetic analysis of CAM-3003 was undertaken to confirm antibody exposure levels between sham- and CS-treated animals in both lung and blood compartments. No difference in antibody exposure was observed between sham- and CS-treated animals (fig. 3b). In vivo anti-GM-CSF and anti-GM-CSFR antibody administration resulted in a comparable and significantly attenuated CSinduced neutrophilic inflammation. Control mice receiving the isotype control antibody demonstrated no attentuation of CSinduced inflammation (fig. $3 \mathrm{c}$ and d). In order to determine changes in neutrophil numbers in the interstitium, flow cytometry of lung single-cell suspensions was undertaken. The percentage of Gr-1-high cells was significantly increased by CS exposure when compared with sham-treated animals $(\mathrm{p}<0.05)$. No significant difference was observed between CSexposed isotype control-, anti-GM-CSF- or anti-GM-CSFRtreated mice (fig. 4).

\section{Anti-GM-CSF ligand and receptor antibodies attenuate CS- induced DC expansion and T-cell activation}

It is widely accepted that GM-CSF promotes DC maturation, a critical step in antigen-driven T-cell responses (reviewed in [4]). Therefore, we investigated whether intervention with antiGM-CSF or receptor antibodies attenuated DC expansion and T-cell activation following CS exposure. Anti-GM-CSF ligand antibody intervention abrogated CS-induced increases in myeloid DCs (mDCs) (CD11c-high MHC II-high) and plasmacytoid DCs (pDCs) (CD11c-high MHC II+ B220+) DCs (fig. 5a and $b$ ). Administration of an anti-GM-CSFR antibody also reduced the frequency of $\mathrm{mDC}$ (fig. $5 c$ and $d$ ), while no effect was observed on pDCs.

Anti-GM-CSF ligand antibody intervention abrogated CSinduced increases in activated CD69+ CD4+ and CD8+ T-cells compared with isotype control-treated animals. In contrast to ligand-blocking antibody, a reduction was only observed for CD69+ CD8+ T-cells, but not CD4+ T cells (fig. 5e and f), in mice treated with an anti-GM-CSFR antibody.

\section{Anti-GM-CSFR antibody treatment reduces IL-12, matrix metalloproteinase 12 mRNA and protein expression in CS- exposed BALB/c mice}

In order to study the mechanisms of CS-induced inflammation, we analysed the expression of lung cytokines and chemokines, as well as matrix metalloproteinases (MMPs), using Fluidigm analysis. CS exposure caused a significant increase in mRNA expression for: the cytokines IL-12p40, IL-1 $\beta$, IL-33, IL-6, mucin $5 \mathrm{AC}$, and TNF- $\alpha$; the chemokines KC, monocyte chemotactic protein-1, C-C motif chemokine ligand (CCL)3, CCL4, CCL9, colony-stimulating factor-3 and CXC motif ligand (CXCL)2; and MMP9 and MMP12 (table 1). Anti-GM-CSFR antibody significantly attenuated the mRNA expression of IL-12p40 (fig. 6a) and MMP-12 (fig. 6b) relative to isotype control antibody treatment (fig. 6 and table 1). No significant difference was observed with TNF- $\alpha$ or CXCL2 relative to isotype control (fig. 6c and d).

In order to confirm the mRNA expression data, we examined expression of cytokines IL-1 $\beta$ KC, IFN- $\gamma$, IL-2, IL-12 and IL-4. Increased expression of IL-1 $\beta, \mathrm{KC}$ and IL-12 was observed in CS-exposed animals injected with isotype antibody control. The anti-GM-CSFR antibody significantly reduced expression of IL-12 in the BAL and lung homogenates, while IL-1 $\beta$ or KC levels remained unchanged. A similar observation was made for IL-1 $\beta$ levels in animals treated with the anti-ligand antibody (supplemental table 1). IFN- $\gamma$, IL-2 and IL-4 levels were all below the limit of detection in sham- and CS-treated groups (table 2).

\section{DISCUSSION}

Neutrophils and macrophages are regularly implicated in the pathogenesis of COPD. It has previously been shown that as the severity of the disease progresses, the proportion of inflammatory cells in the airways increases [2]. Therefore, it has been proposed that interfering with mechanisms that blunt the activity of these cells may provide clinical benefit. Consequently, GM-CSF has been implicated as a potential mediator in COPD.

Whilst GM-CSF is elevated in the lungs from patients with COPD or chronic bronchitis [8], interventions targeting this pathway in models of CS-induced lung inflammation have not been widely described [10]. Here we showed that, following 4 days of CS exposure, we observed a consistent increase in total cells in the BAL, the majority being neutrophils. Moreover, GMCSF was elevated at both the transcript and protein levels in the lungs from these animals. Interestingly, whilst VLAHOS et al. [10] demonstrated attenuation of CS-induced lung inflammation with antibodies to GM-CSF, they were unable to measure GMCSF protein in the BAL. Likewise, they also demonstrated attenuation of neutrophils and macrophages; however, in our model of CS-induced inflammation, we observed an increase and subsequent attenuation of neutrophils only, using either antibody. It is of note that the type of CS-exposure system and route of antibody delivery were different and, thus, may account for the subtle differences observed between this study and that previously described [10].

In order to determine whether the GM-CSF pathway played a significant role in the recruitment of inflammatory cells into the BAL, we evaluated both an anti-GM-CSF neutralising antibody (22E9) and an anti-GM-CSFR $\alpha$-chain antibody (CAM3003) in this model. To rule out the potential of antibody-dependent cellular cytotoxicity, the anti-GM-CSFR antibody was specifically expressed as a mouse IgG1 antibody. Consequently, no evidence of neutrophil depletion in peripheral blood was observed with this antibody (data not shown). Using either approach, we inhibited the recruitment of neutrophils 
in the BAL by dosing via the systemic compartment; however, we did not reduce neutrophil numbers in the tissue, as defined by flow cytometry. This observation in the BAL is consistent with the previous observation that intranasal delivery of the same GM-CSF antibody (22E9) was reported to blunt CSinduced neutrophil and macrophage recruitment in the BAL $[10,23]$. Whilst we did not observe a significant increase in macrophages and lymphocytes upon CS exposure, we did observe a modest but significant change in the percentage of mature lung DCs that was attenuated with either antibody. In vitro, it has been shown that diesel exhaust particle-conditioned medium from epithelial cells can induce a GM-CSF-dependent DC maturation [24], suggesting that CS exposure may also contribute to DC maturation in a similar manner. This is supported by the observation that, in turn, both CD4+ and CD8+ T-cells increase CD69 expression following CS exposure as previously described [21], and that this was partially inhibited by blockade of the GM-CSF pathway. Interestingly only CD8+ T-cell activation was suppressed by both mechanisms, but the CD4+ CD69+ activated T-cells were not affected by treatment with anti-GM-CSFR. The reason for this is unclear and merits further investigation. Nevertheless, T-cells do not express the GM-CSFR $\alpha$-chain; therefore, these data suggest that a reduction in the CS-induced T-cell activation maybe an indirect effect of GM-CSF stimulation of myeloid cells rather than a direct effect of GM-CSF on T-cells.

Recent studies [22] have shown that MyD88-, Toll-like receptor 4- and IL-1R-deficient mice all had reduced lung neutrophilia and cytokine levels in the lung following CS exposure. Therefore, we investigated GM-CSF expression in CS-exposed IL-1R-deficient mice. In this system, we confirmed that
CS-induced neutrophilia was suppressed and that KC mRNA levels were significantly inhibited (data not shown). Based on our observations, it may be concluded that GM-CSF plays a central role in CS lung inflammation, potentially downstream of the IL-1R pathway. A similar observation has also been observed in a mouse model of IL-1 $\beta$-driven monoarthitis [25].

In order to determine the mechanism of GM-CSF blockade, we investigated the change in expression levels of various proteins and mRNAs in the lung. Whilst CS exposure enhanced the expression of a range of cytokines, blocking the GM-CSF pathway did not appear to suppress cytokines typically associated with neutrophilic inflammation, such as IL-1 $\beta$ and KC. GM-CSF is known to activate neutrophils, promoting adhesion in pulmonary vascular endothelium [26], recruitment [27] and sensitisation to chemokines [28]. The lack of a direct effect on KC, IL-1 $\beta$ and TNF suggests that GM-CSF may operate downstream of these cytokines, potentially rendering neutrophils less responsive to the direct effect of these molecules, and may also account for there being no apparent difference between the percentage of neutrophils in lung homogenates from CS-exposed mice treated with either an anti-ligand antibody or anti-receptor antibody. It has also been demonstrated that GM-CSF promotes neutrophil survival by preventing apoptosis [29] and, thus, GM-CSF blockade may also have enhanced neutrophil apoptosis and clearance by macrophages. Further studies are warranted to better understand how neutrophil numbers in the BAL are attenuated and the activation status of resident neutrophils within the lung.

However, inhibition of the GM-CSF pathway during CS exposure did inhibit IL-12p40 and IL-12 production at the transcript and protein level, respectively. In vitro, GM-CSF has

TABLE 1 Fluidigm mRNA expression analysis of 4-day cigarette smoke (CS)-exposed BALB/c mice injected with anti-granulocyte-macrophage colony-stimulating factor receptor (GM-CSFR) antibody

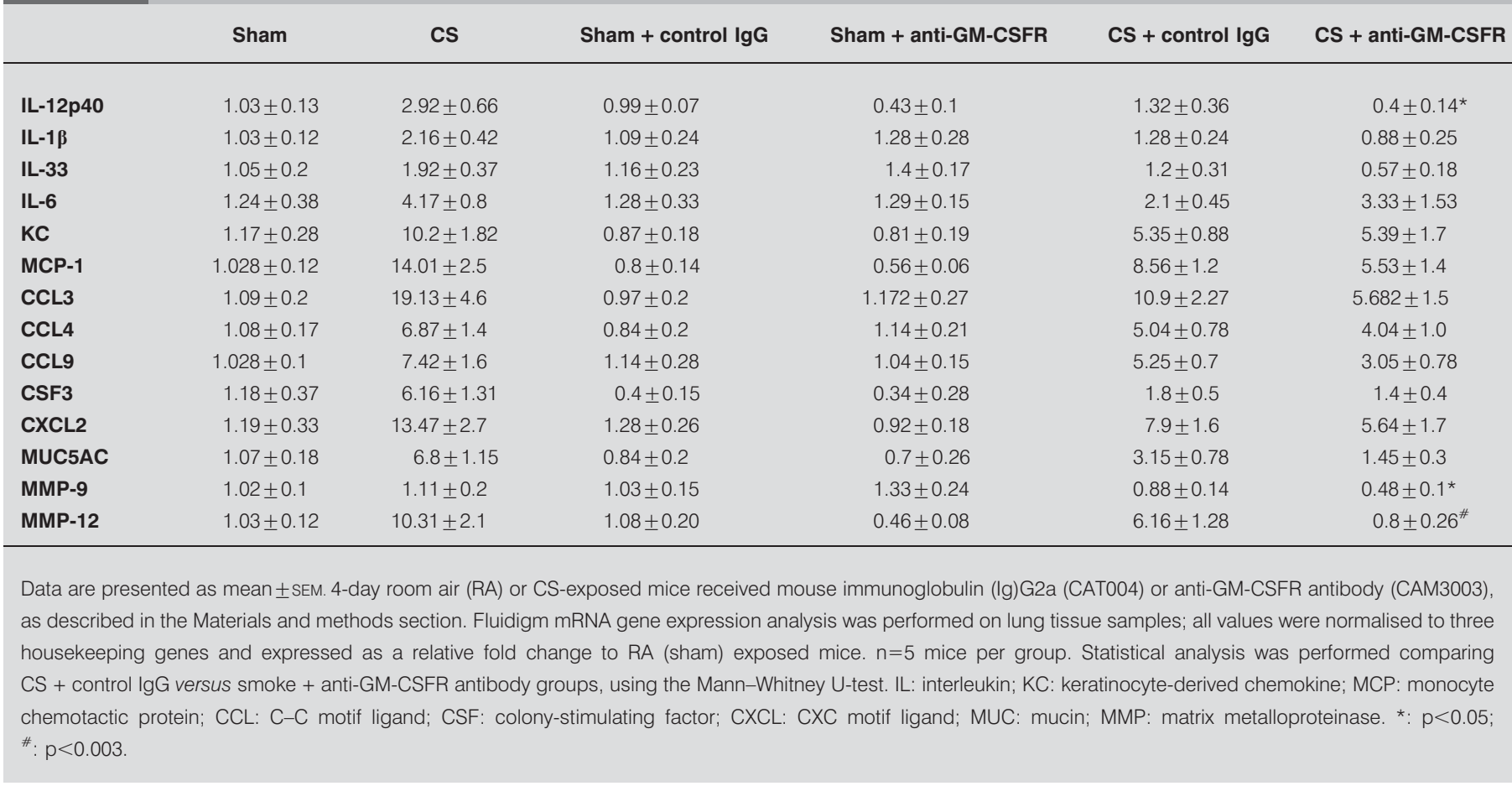



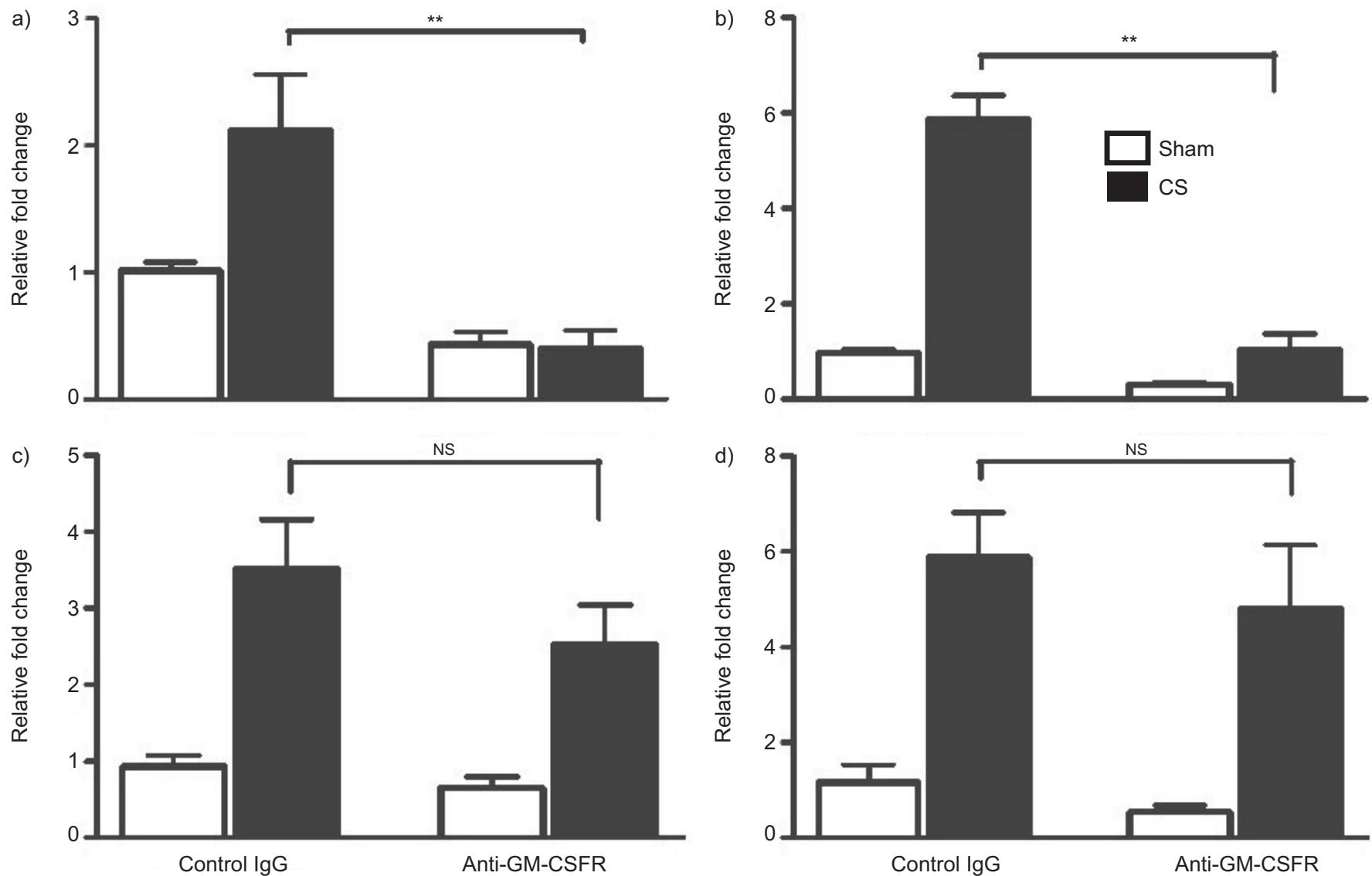

FIGURE 6. a) Interleukin-12 p40, b) matrix metalloproteinase 12, c) tumour necrosis factor- $\alpha$ and d) CXC ligand 2 mRNA expression. BALB/c mice were exposed to room air (sham) or cigarette smoke (CS) for 4 days. Mice were injected i.p. with anti-granulocyte-macrophage colony-stimulating factor receptor (GM-CSFR) or isotype control antibodies. All values were normalised to three housekeeping genes and expressed as the relative fold change over sham-exposed mice. Bars represent means and whiskers represent standard errors of the mean. $\mathrm{n}=5$ per group. Statistical analysis was performed comparing CS-exposed control immunoglobulin (Ig)G versus anti-GM-CSFR antibody-treated mice using the Mann-Whitney U-test. NS: nonsignificant. **: $p<0.001$

previously been shown to induce IL-12 from both DCs and macrophages [30]. In addition, mice deficient in GM-CSF exposed either to a viral [31] or bacterial [32] pathogen demonstrated a reduction in IL-12 production. Furthermore, overexpression of GM-CSF in the lungs of normal mice stimulates an increase in IL-12 production [33]. Similarly, we showed that IL-12p40 was upregulated in the lungs of CSexposed mice and that these levels were attenuated with either antibody, indicating that IL-12p40 expression is downstream of CS-induced GM-CSF production. Chronic CS exposure in mice plus the addition of virus or viral mimetics (polyI:C) has been shown to promote an increase in IL-12p40 production [34];

TABLE 2 Protein expression following room air (sham) or subchronic 4-day cigarette smoke (CS) exposure with or without i.p. anti-granulocyte-macrophage colony-stimulating factor receptor (GM-CSFR) antibody

\begin{tabular}{|c|c|c|c|c|c|c|}
\hline & \multicolumn{3}{|c|}{ BAL fluid } & \multicolumn{3}{|c|}{ Lung homogenate } \\
\hline & Sham & CS + control lgG & CS + anti-GM-CSFR & Sham & CS + control lgG & CS + anti-GM-CSFR \\
\hline $\mathrm{IL}-1 \beta \mathrm{pg} \cdot \mathrm{mL}^{-1}$ & $3 \pm 1$ & $46 \pm 4^{\#}$ & $35 \pm 5$ & $132 \pm 32$ & $508 \pm 65^{\#}$ & $419 \pm 40$ \\
\hline $\mathrm{KC} \mathrm{pg} \cdot \mathrm{mL}^{-1}$ & $112 \pm 28$ & $434 \pm 56^{\#}$ & $420 \pm 55$ & $257 \pm 26$ & $1616 \pm 90^{\#}$ & $2143 \pm 123$ \\
\hline $\mathrm{IL}-12 \mathrm{pg} \cdot \mathrm{mL}^{-1}$ & $133 \pm 33$ & $1805 \pm 208^{\#}$ & $653 \pm 112^{\bullet}$ & $753 \pm 63$ & $2656 \pm 18^{\#}$ & $1665 \pm 117^{\bullet}$ \\
\hline
\end{tabular}

Data are presented as mean \pm SEM. Protein levels were determined using MesoScaleDevices multiarray platform, using the murine pro-inflammatory and T-helper cell (Th) type 1/Th2 cytokine panels. $n=8-10$ mice per group from two independent experiments. Statistical analysis was performed using the Mann-Whitney U-test. BAL: bronchoalveolar lavage; Ig: immunoglobulin; IL: interleukin; KC: keratinocyte-derived chemokine. ${ }^{\#:}: p<0.0001$ comparing sham- versus CS-exposed, control lgG-treated mice; ": p $<0.0005$ comparing CS-exposed, control IgG- versus anti-GM-CSFR-treated mice. 
however, this is the first time that this elevation has been shown in such an subchronic model. Nevertheless, relatively little is known about the expression of this molecule and its role in COPD [24] and, therefore, further studies are warranted.

In addition to an upregulation of IL-12p40, subchronic smoke exposure also stimulated an increase in MMP-12 mRNA, consistent with previous reports [35]. Tissue remodelling and proteolytic damage are hallmarks of COPD, and proteases, such as MMP9 and MMP12, have been shown to be elevated within the COPD lung $[36,37]$. Furthermore, mice deficient in MMP-12 do not present with an emphysematous phenotype following chronic smoke exposure [38]. Both neutrophils and macrophages are known to be a rich source of MMP-12 [39] and their numbers correlate with disease severity in COPD [40]. In another model of CS induced lung inflammation, macrophages were described as being the main source of this protease [35]. In the present study, we have demonstrated that blocking GM-CSF signalling attenuated MMP-12 expression in lung tissue. These data are consistent with the observation that GM-CSF can induce MMP-12 production from human peripheral blood monocyte-derived macrophages [41, 42]. Interestingly, whilst we did observe a change in MMP-12 mRNA levels in the lung, as recently described [10], we did not observe a statistically significant change in either TNF- $\alpha$ mRNA or macrophage inflammatory protein $2 \alpha$ (fig. $6 \mathrm{c}$ and $\mathrm{d}$ ) in this system, suggesting that the route of delivery may have subtly different effects on mRNA profiles within the lung. This difference was not due to antibody exposure, as pharmacokinetic analysis of CAM-3003 confirmed significant levels of antibody in both serum and lung homogenates (fig. 3b).

In conclusion, the present study reports that systemic delivery of anti-GM-CSF or anti-GM-CSFR antibodies attenuates CSinduced neutrophilia. Furthermore, GM-CSF appears to play a role in activating resident lung DCs and lymphocytes, providing a link between the initial innate response to subchronic CS exposure and supporting the adaptive response. Further studies are warranted to characterise this relationship and shed light on the potential role of this cytokine in COPD.

\section{SUPPORT STATEMENT}

J.K. Nikota was supported by Ontario's Early Research Award program (ERA). M.R. Stämpfli holds a Canadian Institutes for Health Research New Investigator award.

\section{STATEMENT OF INTEREST}

Statements of interest for N.H.E. Davis, E.S. Cohen, I.K. Anderson, A.J. Coyle, R. Kolbeck, A.A. Humbles, M.R. Stämpfli and M.A. Sleeman can be found at www.erj.ersjournals.com/site/misc/statements.xhtml

\section{ACKNOWLEDGEMENTS}

The authors gratefully acknowledge the expert technical support of J. Kasinska and S. Kianpour (both McMaster University, Hamilton, ON, Canada), J. Elvin (MedImmune Ltd, Cambridge, UK), P. Brohawn and A. Keller (both MedImmune LLC, Gaithersburg, MD, USA), and the Hybridoma team at MedImmune (Cambridge).

\section{REFERENCES}

1 Patel RR, Ryu JH, Vassallo R. Cigarette smoking and diffuse lung disease. Drugs 2008; 68: 1511-1527.
2 Hogg JC. Pathophysiology of airflow limitation in chronic obstructive pulmonary disease. Lancet 2004; 364: 709-721.

3 Pesci A, Majori M, Cuomo A, et al. Neutrophils infiltrating bronchial epithelium in chronic obstructive pulmonary disease. Respir Med 1998; 92: 863-870.

4 Hamilton JA. Colony-stimulating factors in inflammation and autoimmunity. Nat Rev Immunol 2008; 8: 533-544.

5 Trapnell BC, Whitsett JA. Gm-CSF regulates pulmonary surfactant homeostasis and alveolar macrophage-mediated innate host defense. Annu Rev Physiol 2002; 64: 775-802.

6 Bozinovski S, Jones JE, Vlahos R, et al. Granulocyte/macrophagecolony-stimulating factor (GM-CSF) regulates lung innate immunity to lipopolysaccharide through Akt/Erk activation of $\mathrm{NF \kappa B}$ and AP-1 in vivo. J Biol Chem 2002; 277: 42808-42814.

7 Balbi B, Bason C, Balleari E, et al. Increased bronchoalveolar granulocytes and granulocyte/macrophage colony-stimulating factor during exacerbations of chronic bronchitis. Eur Respir J 1997; 10: 846-850.

8 Saha S, Doe C, Mistry V, et al. Granulocyte macrophage colony stimulating factor expression in induced sputum and bronchial mucosa in asthma and COPD. Thorax 2009; 64: 671-676.

9 Tsoumakidou M, Tzanakis N, Chrysofakis G, et al. Nitrosative stress, heme oxygenase-1 expression and airway inflammation during severe exacerbations of COPD. Chest 2005; 127: 1911-1918.

10 Vlahos R, Bozinovski S, Chan SP, et al. Neutralizing granulocyte/ macrophage colony-stimulating factor inhibits cigarette smokeinduced lung inflammation. Am J Respir Crit Care Med 2010; 182: 34-40.

11 Hansen G, Hercus TR, McClure BJ, et al. The structure of the GMCSF receptor complex reveals a distinct mode of cytokine receptor activation. Cell 2008; 134: 496-507.

12 Hercus TR, Thomas D, Guthridge MA, et al. The granulocytemacrophage colony-stimulating factor receptor: linking its structure to cell signaling and its role in disease. Blood 2009; 114: 1289-1298.

13 Shapiro JA, Jacobs EJ, Thun MJ. Cigar smoking in men and risk of death from tobacco-related cancers. J Natl Cancer Inst 2000; 92: 333-337.

14 Churg A, Cosio M, Wright JL. Mechanisms of cigarette smokeinduced COPD: insights from animal models. Am J Physiol Lung Cell Mol Physiol 2008; 294: L612-L631.

15 Vlahos R, Bozinovski S, Gualano RC, et al. Modelling COPD in mice. Pulm Pharmacol Ther 2006; 19: 12-17.

16 Sethi G, Sung B, Kunnumakkara AB, et al. Targeting TNF for treatment of cancer and autoimmunity. Adv Exp Med Biol 2009; 647: 37-51.

17 Maker AV, Attia P, Rosenberg SA. Analysis of the cellular mechanism of antitumor responses and autoimmunity in patients treated with CTLA-4 blockade. J Immunol 2005; 175: 7746-7754.

18 Ohsugi Y. Recent advances in immunopathophysiology of interleukin-6: an innovative therapeutic drug, tocilizumab (recombinant humanized anti-human interleukin-6 receptor antibody), unveils the mysterious etiology of immune-mediated inflammatory diseases. Biol Pharm Bull 2007; 30: 2001-2006.

19 Nopp A, Johansson SG, Adédoyin J, et al. After 6 years with Xolair; a 3-year withdrawal follow-up. Allergy 2010; 65: 56-60.

20 Church LD, McDermott MF. Canakinumab, a fully-human mAb against IL-1 $\beta$ for the potential treatment of inflammatory disorders. Curr Opin Mol Ther 2009; 11: 81-89.

21 Botelho FM, Gaschler GJ, Kianpour S, et al. Innate immune processes are sufficient for driving cigarette smoke-induced inflammation in mice. Am J Respir Cell Mol Biol 2010; 42: 394-403.

22 Doz E, Noulin N, Boichot E, et al. Cigarette smoke-induced pulmonary inflammation is TLR4/MyD88 and IL-1R1/MyD88 signaling dependent. J Immunol 2008; 180: 1169-1178.

23 Vlahos R, Bozinovski S, Hamilton JA, et al. Therapeutic potential of treating chronic obstructive pulmonary disease (COPD) by neutralising granulocyte macrophage-colony stimulating factor (GM-CSF). Pharmacol Ther 2006; 112: 106-115. 
24 Bleck B, Tse DB, Jaspers I, et al. Diesel exhaust particle-exposed human bronchial epithelial cells induce dendritic cell maturation. J Immunol 2006; 176: 7431-7437.

25 Yang YH, Hamilton JA. Dependence of interleukin-1-induced arthritis on granulocyte-macrophage colony-stimulating factor. Arthritis Rheum 2001; 44: 111-119.

26 Yong KL, Rowles PM, Patterson KG, et al. Granulocyte-macrophage colony-stimulating factor induces neutrophil adhesion to pulmonary vascular endothelium in vivo: role of beta 2 integrins. Blood 1992; 80: 1565-1575.

27 Gomez-Cambronero J, Horn J, Paul CC, et al. Granulocytemacrophage colony-stimulating factor is a chemoattractant cytokine for human neutrophils: involvement of the ribosomal p70 S6 kinase signaling pathway. J Immunol 2003; 171: 6846-6855.

28 Shen L, Fahey JV, Hussey SB, et al. Synergy between IL-8 and GMCSF in reproductive tract epithelial cell secretions promotes enhanced neutrophil chemotaxis. Cell Immunol 2004; 230: 23-32.

29 Coxon A, Tang T, Mayadas TN. Cytokine-activated endothelial cells delay neutrophil apoptosis in vitro and in vivo. A role for granulocyte/macrophage colony-stimulating factor. I Exp Med 1999; 190: 923-934.

30 Trinchieri G, Gerosa F. Immunoregulation by interleukin-12. J Leukoc Biol 1996; 59: 505-511.

31 Berclaz P-Y, Shibata Y, Whitsett JA, et al. GM-CSF, via PU.1, regulates alveolar macrophage $\mathrm{F} c \gamma \mathrm{R}$-mediated phagocytosis and the IL-18/IFN- $\gamma$-mediated molecular connection between innate and adaptive immunity in the lung. Blood 2002; 100: 4193-4200.

32 Coon C, Beagley KW, Bao S. The role of granulocyte macrophagecolony stimulating factor in gastrointestinal immunity to salmonellosis. Scand J Immunol 2009; 70: 106-115.

33 Bukreyev A, Belyakov IM, Berzofsky JA, et al. Granulocytemacrophage colony-stimulating factor expressed by recombinant respiratory syncytial virus attenuates viral replication and increases the level of pulmonary antigen-presenting cells. J Virol 2001; 75: 12128-12140.

34 Kang M, Lee C, Lee J, et al. Cigarette smoke selectively enhances viral PAMP- and virus-induced pulmonary innate immune and remodeling responses in mice. J Clin Invest 2008; 118: 2771-2784.

35 Vlahos R, Bozinovski S, Jones JE, et al. Differential protease, innate immunity, and NF-kappaB induction profiles during lung inflammation induced by subchronic cigarette smoke exposure in mice. Am J Physiol Lung Cell Mol Physiol 2006; 290: L931-L945.

36 Imai K, Dalal SS, Chen ES, et al. Human collagenase (matrix metalloproteinase-1) expression in the lungs of patients with emphysema. Am J Respir Crit Care Med 2001; 163: 786-791.

37 Montaño M, Beccerril C, Ruiz V, et al. Matrix metalloproteinases activity in COPD associated with wood smoke. Chest 2004; 125: 466-472.

38 Hautamaki RD, Kobayashi DK, Senior RM, et al. Requirement for macrophage elastase for cigarette smoke-induced emphysema in mice. Science 1997; 277: 2002-2004.

39 Ilumets H, Rytilä P, Demedts I, et al. Matrix metalloproteinases -8, -9 and -12 in smokers and patients with stage 0 COPD. Int J Chron Obstruct Pulmon Dis 2007; 2: 369-379.

40 Babusyte A, Stravinskaite K, Jeroch J, et al. Patterns of airway inflammation and MMP-12 expression in smokers and ex-smokers with COPD. Respir Res 2007; 8: 81.

$41 \mathrm{Wu} \mathrm{L}$, Fan J, Matsumoto Si, et al. Induction and regulation of matrix metalloproteinase- 12 by cytokines and CD40 signaling in monocyte/macrophages. Biochem Biophys Res Commun 2000; 269: 808-815.

$42 \mathrm{Wu}$ L, Tanimoto A, Murata $\mathrm{Y}$, et al. Induction of human matrix metalloproteinase-12 gene transcriptional activity by GM-CSF requires the AP-1 binding site in human U937 monocytic cells. Biochem Biophys Res Commun 2001; 285: 300-307. 